\title{
Influence of timing of intraaortic balloon placement in cardiac surgical patients
}

\author{
Jayshree D. Lavana, MBBS, MD, FJFICM, John F. Fraser, MB ChB, MRCP, FRCA, FFARCSI, FJFICM, \\ Susan E. Smith, BSc (Hons), Lesley Drake, BBusHA (HIM), Peter Tesar, MBBS, FRCS, and \\ Daniel V. Mullany, MBBS, FANZCA, FJICM
}

Objective: The study objective was to evaluate the association between timing of intraaortic balloon pump insertion and outcomes in patients undergoing cardiac surgery.

\begin{abstract}
Methods: All patients aged 18 years or more who underwent coronary artery bypass surgery, cardiac valve surgery, or thoracic aortic surgery between January 2002 and December 2007 were included. Data were obtained from cardiac surgery and intensive care databases. Patients were categorized as receiving a preoperative, intraoperative, or postoperative intraaortic balloon pump and compared with a reference group who did not receive an intraaortic balloon pump. Summary and descriptive statistics were used to compare the groups. Logistic regression was used to model in-hospital mortality, and survival methods were used to model time to event data, such as length of stay.
\end{abstract}

Results: There were 7440 patients included over a 6-year period, of whom $217(2.9 \%)$ received a preoperative intraaortic balloon pump, $184(2.4 \%)$ received an intraoperative intraaortic balloon pump, and $42(0.56 \%)$ received a postoperative intraaortic balloon pump. Logistic European System for Cardiac Operative Risk Evaluation-derived predicted risk of death was higher across all intraaortic balloon pump groups compared with the group with no intraaortic balloon pump. Observed in-hospital mortality was significantly lower in the preoperative group $(10 \%)$ and the group with no intraaortic balloon pump $(0.8 \%)$ compared with the intraoperative $(16 \%)$ and postoperative $(29 \%)$ groups. Risk-adjusted mortality was also lower in the preoperative group.

Conclusion: This study comparing outcomes in patients undergoing cardiac surgical procedures with timing of intraaortic balloon pump placement revealed that the use of preoperative intraaortic balloon pumps was associated with a strong trend toward reduction in in-hospital mortality despite a higher predicted mortality in this group. The study provides support to the growing body of literature advocating preoperative use of intraaortic balloon pumps in carefully selected patients. (J Thorac Cardiovasc Surg 2010;140:80-5)

The intraaortic balloon pump (IABP) is the most commonly used mechanical support method in cardiac surgery. It improves diastolic coronary blood flow, reduces left ventricular afterload, and enhances endomyocardial perfusion. IABPs are reported to be used in $5 \%$ to $10 \%$ of cardiac surgical cases. ${ }^{1,2}$ Insertion practices vary, with the Benchmark Registry and Society of Thoracic Surgeons database showing that $52.4 \%$ and $63.5 \%$, respectively, are inserted preoperatively. ${ }^{3,4}$ Practices in Europe differ from the United States, with approximately $40 \%$ to $45 \%$ inserted preoperatively according to a large European study. ${ }^{5}$ In our center, we have seen an increasing use of IABPs over time. ${ }^{6,7}$ Furthermore,

From the Critical Care Research Group, Departments of Intensive Care and Cardiac Surgery, The Prince Charles Hospital, Brisbane, Queensland, Australia.

Disclosures: None.

Received for publication June 19, 2009; revisions received Aug 20, 2009; accepted for publication Sept 17, 2009; available ahead of print Nov 12, 2009.

Address for reprints: Jayshree D. Lavana, MBBS, MD, FJFICM, Critical Care Research Group, John B. McCarthy Intensive Care Unit, The Prince Charles Hospital, Rode Road, Chermside, Queensland, Australia 4032 (E-mail: jayshree_lavana@health.qld.gov.au).

0022-5223/\$36.00

Crown Copyright (C) 2010 Published by Elsevier Inc. on behalf of The American Association for Thoracic Surgery

doi:10.1016/j.jtcvs.2009.09.033 significant differences between Australian and European cardiac surgical populations and lack of local, large studies make a formal evaluation of preoperative IABP utility imperative. $^{8}$

The role of the preoperative prophylactic IABP is subject to debate. Dyub and colleagues ${ }^{9}$ recently performed a meta-analysis of randomized controlled trials and cohort studies that showed lower mortality and hospital length of stay in the preoperative group. The largest and only multicenter study in that analysis showed no survival advantage with preoperative insertion in stable patients undergoing coronary artery bypass surgery versus intraoperative insertion as required, although postoperative length of hospital stay was reduced in the preoperative insertion cohort. ${ }^{10}$ The inclusion and exclusion criteria vary between studies, limiting generalizability, and the authors discussed limitations and potential biases of the study, including small patient numbers, lack of multicenter studies, and variability in criteria for insertion.

It is widely recognized that the cardiac surgical patient is undergoing more complex surgery and that the incidence of isolated coronary artery bypass grafting $(\mathrm{CABG})$ is decreasing, whereas that of combined valve plus CABG surgery is increasing. Controversy still exists as to the optimal timing 


\section{Abbreviations and Acronyms}

$$
\begin{array}{ll}
\text { CABG } & =\text { coronary artery bypass grafting } \\
\text { EuroSCORE } & =\text { European System for Cardiac } \\
& \text { Operative Risk Evaluation } \\
\text { IABP } & \text { intraaortic balloon pump } \\
\text { ICU } & \text { intensive care unit }
\end{array}
$$

of IABP insertion, with difficulty in predicting who will require an IABP and who can be managed without one. Concerns remain surrounding the increased use of preoperative IABPs, because complications, although rare, may be lifethreatening. These can include major vascular, limb ischemia, and bleeding complications. ${ }^{11}$

In light of these factors, the aim of this study was to review the timing of IABP insertion and outcomes in an unselected cardiac surgical sample. We analyzed outcomes adjusting for known risk factors and determined clinical and operative risk factors for intraoperative and postoperative insertion.

\section{MATERIALS AND METHODS}

This study was performed at The Prince Charles Hospital, a 450-bed tertiary referral, university-affiliated, teaching hospital with a predominantly cardiothoracic patient case mix. Data were obtained from the cardiac surgery and intensive care databases. The patients included were those who underwent coronary artery bypass surgery, cardiac valve surgery, or aortic surgery between January 2002 and December 2007. Patients were excluded if they were aged less than 18 years or were undergoing transplant, ventricular assist device, pulmonary thromboendarterectomy, or adult congenital heart surgery. Data were collected by trained data collectors, and data quality was maintained by several processes, including edit checks (built into the database) and scheduled data cleaning. All cardiac surgeons were sent a report on surgical outcomes for their individual patients before each monthly audit for confirmation of accuracy and completeness. All missing fields were treated as the variable being absent, and no imputations were used.

The primary outcome measure was in-hospital mortality. Secondary outcome measures were duration of ventilation in the intensive care unit (ICU), length of stay in the ICU, and length of hospital stay.

Patients were categorized depending on the timing of IABP placement. The control group included patients who did not receive an IABP. The preoperative group included patients who had an IABP placed before surgical skin incision. The intraoperative group included patients in whom the IABP was placed after skin incision but before leaving the operating room. The postoperative group included patients who received an IABP after leaving the operating room of the primary operation. Preoperative IABPs were usually placed in the pre-theater setting (cardiac catheterization laboratory, interhospital transfer, or intensive care in rare cases), and the common institutional indications were critical left main stem coronary disease, cardiogenic shock after acute myocardial infarction or unstable angina, mechanical complications of acute myocardial infarction (ie, acute mitral regurgitation, and ventricular septal defect).

The decision to place an IABP intraoperatively or postoperatively was at the discretion of the operating surgeon. Common indications were difficulty or inability to separate from bypass and unanticipated intraoperative or postoperative complications.

Data collected included demographic, clinical, and outcome data. Unless otherwise specified, variables were defined as used in the European System for Cardiac Operative Risk Evaluation (EuroSCORE) ${ }^{12}$ Clinical and demographic variables were compared across all patient categories, with the Kruskal-Wallis test for continuous variables and the chi-square test of general association for categoric variables. Risk adjustment for outcomes used preoperative and intraoperative variables. Potential confounding exists because EuroSCORE defines critical preoperative state as a requirement for preoperative balloon pump. Because this is potentially a subjective decision, analysis was preformed with and without this variable as a risk factor.

The association between timing of IABP insertion and in-hospital mortality was modeled using logistic regression, controlling for known predictors of risk in cardiac surgery. A second model was developed to determine predictors of the need for an intraoperative or postoperative IABP using preoperative variables. Standard diagnostic methods were used to assess model performance with analysis of calibration and discrimination. Test of trend was applied to determine the trend of IABP use over the 6-year study period. No funding agency was involved in the study, and administrative staff support was provided by Critical Care Research Group. The study received approval of the Ethics Committee of the institution before its conduct.

\section{RESULTS}

There were 7440 patients included in the study, of whom $443(5 \%)$ received an IABP. The characteristics of the sample are shown in Table 1 . In this sample, 217 patients $(2.9 \%)$ received a preoperative IABP, 184 patients $(2.5 \%)$ received an intraoperative IABP, and 42 patients $(0.6 \%)$ received a postoperative IABP. Compared with the intraoperative or postoperative group, the preoperative group was more likely to undergo CABG only, undergo emergency surgery, be in a critical preoperative condition, and have preoperative unstable angina or a recent myocardial infarction. The intraoperative group was more likely to undergo more complex surgery and reoperation surgery, and have vascular disease and pulmonary hypertension. There was no difference between the groups with regard to age and ventricular function. A greater percentage of female patients received postoperative IABPs. Female gender and vascular disease are known higher risk factors for ischemic limb complications and may reflect the practice of deferring or avoiding "prophylactic" IABP placement in high-risk candidates until it becomes clear it is clinically indicated (eg, to separate from cardiopulmonary bypass). Table 1 shows that most patients with left main coronary disease $(1265 / 1428=86 \%)$, 3-vessel coronary disease $(3697 / 3892=95 \%)$, poor ejection fraction $(163 / 249=65 \%)$, unstable angina $(152 / 238=64 \%)$, or reoperation $(759 / 832=91 \%)$ did not receive an IABP.

The changes in practice over time are shown in Table 2. During the study period, there was a trend to increased preoperative and total IABP use (test for trend $P<.01$ ), the exception being years 2004 and 2005, when intraoperative and postoperative use was higher compared with other years, but declined again subsequently. This could be attributed to changes in surgical personnel or the preferences of the operating surgeon/s at these time points.

Table 3 shows the outcomes during the study period. The overall mortality was $1.6 \%$ : $0.8 \%$ in the control group, $9.7 \%$ in the preoperative group, $15.8 \%$ in the intraoperative 
TABLE 1. Patient characteristics by timing of intraaortic balloon pump insertion

\begin{tabular}{|c|c|c|c|c|}
\hline & No IABP N $=6997$ & Preoperative $\mathbf{N}=217$ & Intraoperative $N=184$ & Postoperative $\mathrm{N}=42$ \\
\hline \multicolumn{5}{|l|}{ Variable } \\
\hline Mean age (SD y) & $64(12)$ & $65(11)$ & $66(11)$ & $65(13)$ \\
\hline Gender (female \%) & 1995 (29) & $58(27)$ & $49(27)$ & $19(45)$ \\
\hline Serum creatinine $(\mathrm{SD}, \mu \mathrm{mol} / \mathrm{L})$ & $100(50)$ & $120(98)$ & $120(61)$ & $130(72)$ \\
\hline Creatinine $>200 \mu \mathrm{mol} / \mathrm{L}(\%)$ & $169(2.4)$ & $19(9)$ & $9(5)$ & $8(19)$ \\
\hline Vascular disease $(\%)$ & $960(14)$ & $19(9)$ & $33(18)$ & $7(17)$ \\
\hline Chronic pulmonary disease $(\%)$ & $957(14)$ & $41(19)$ & $35(19)$ & $9(21)$ \\
\hline Neurological dysfunction (\%) & $108(2)$ & $7(3)$ & $5(3)$ & $2(5)$ \\
\hline Ejection fraction < 30\% (\%) & $163(2)$ & $43(20)$ & $39(21)$ & $4(10)$ \\
\hline Ejection fraction $30 \%-50 \%(\%)$ & $1296(19)$ & $73(34)$ & $60(33)$ & $16(38)$ \\
\hline Critical preoperative state $(\%)$ & $86(1)$ & $202(93)$ & $17(9)$ & $5(12)$ \\
\hline Unstable angina $(\%)$ & $152(2)$ & $52(24)$ & $20(11)$ & $4(10)$ \\
\hline Recent MI $(\%)$ & $1428(20)$ & $153(71)$ & $73(40)$ & $13(31)$ \\
\hline Elective $(\%)$ & $4571(65)$ & $9(4)$ & $74(40)$ & $17(40)$ \\
\hline Urgent $(\%)$ & $2321(33)$ & $110(51)$ & $94(51)$ & $20(48)$ \\
\hline Emergency or salvage surgery $(\%)$ & $105(2)$ & $98(45)$ & $16(9)$ & $3(12)$ \\
\hline Reoperation $(\%)$ & $759(11)$ & $8(4)$ & $56(30)$ & $9(21)$ \\
\hline Endocarditis (\%) & $144(2)$ & $3(1)$ & $8(4)$ & $3(7)$ \\
\hline Pulmonary hypertension (\%) & $378(5)$ & $18(8)$ & $27(15)$ & $8(19)$ \\
\hline Post-infarct VSD (\%) & $1(0.01)$ & $6(3)$ & $2(1)$ & $0(0)$ \\
\hline Left main $>50 \%(\%)$ & $1265(18)$ & $111(51)$ & $42(23)$ & $10(24)$ \\
\hline 3 vessels $(\%)$ & $3697(53)$ & $161(74)$ & $11(65)$ & $23(55)$ \\
\hline 2 vessels $(\%)$ & $1184(17)$ & $39(18)$ & $26(14)$ & $8(19)$ \\
\hline 1 vessel $(\%)$ & $518(7)$ & $8(4)$ & $8(4)$ & 0 \\
\hline No coronary disease $(\%)$ & $1598(23)$ & $9(4)$ & $31(17)$ & $10(26)$ \\
\hline Preoperative ventilation ( $\%$ ) & $40(0.6)$ & $30(14)$ & $4(2)$ & $2(5)$ \\
\hline Logistic EuroSCORE risk of death $(\%)$ & 6.4 & 23.6 & 16.1 & 17 \\
\hline Aortic wall $(\%)$ & $409(6)$ & 0 & $13(7)$ & $4(10)$ \\
\hline CABG only $(\%)$ & $4202(60)$ & $159(73)$ & $83(45)$ & $19(45)$ \\
\hline 1 valve $(\%)$ & $1133(16)$ & $8(4)$ & $12(7)$ & $4(10)$ \\
\hline 1 valve + CABG $(\%)$ & $789(11)$ & $33(15)$ & $44(24)$ & $6(14)$ \\
\hline Aortic or complex surgery $(\%)^{*}$ & $873(12)$ & $17(8)$ & $45(24)$ & $13(31)$ \\
\hline
\end{tabular}

$\overline{C A B G}$, Coronary artery bypass grafting; EuroSCORE, European System for Cardiac Operative Risk Evaluation; IABP, intraaortic balloon pump; $M I$, myocardial infarction; SD, standard deviation; $V S D$, ventricular septal defect. Figures in brackets indicate percentages unless indicated otherwise. ${ }^{*}$ Complex surgery is defined as surgery on 2 or more cardiac valves with or without CABG. Aortic surgery is any surgery involving ascending aorta, aortic arch, or proximal descending thoracic aorta.

group, and $28.6 \%$ in the postoperative group. The cardiopulmonary bypass times were substantially longer in the intraoperative group. Intensive care, postoperative, and mechanical ventilation times were longer in the intraoperative and postoperative groups. When risk adjusted, the inhospital mortality was significantly lower in the preoperative and the "no IABP"' group than the predicted logistic EuroSCORE risk of death (preoperative $9.7 \%$ vs $23.6 \%$; no IABP $0.8 \%$ vs $6.4 \%$ ). Although it was comparable to the predicted mortality in the intraoperative group $(15.8 \%$ vs $16.1 \%$ ), the postoperative group had a higher than predicted mortality ( $28.6 \%$ vs $17 \%)$.

Table 4 shows the univariate and multivariate logistic regression models for in-hospital mortality. The unadjusted mortality shows that preoperative insertion has a lower mortality than intraoperative insertion. When controlling for risk of death using EuroSCORE, preoperative insertion was associated with a significantly lower mortality compared with intraoperative and postoperative insertion. The odds ratio for death is $0.3(0.15-0.61)$ for preoperative compared with intraoperative insertion. The effect is smaller, but there is a trend to reduced mortality when a locally derived risk adjustment model is used. The odds ratio for death is 0.51 (0.23-1.1) for preoperative compared with intraoperative insertion. When cardiopulmonary bypass time is included, there is no statistically significant difference between preoperative and intraoperative insertion. Postoperative insertion remains associated with increased mortality regardless of the risk adjustment method.

The strongest overall predictor was cardiopulmonary bypass time with an odds ratio of 1.01 (95\% confidence interval, 1.01-1.02) per minute. Cardiopulmonary bypass time indicates total combined bypass time, including multiple attempts to separate from bypass. When cardiopulmonary bypass time was included, reoperation, poor left ventricular function, increased creatinine, recent myocardial infarction, 
TABLE 2. Trends in timing of intraaortic balloon bump insertion over the past 6 years

\begin{tabular}{lccccc}
\hline Year & No IABP & Preoperative (\%) & Intraoperative (\%) & Postoperative (\%) & IABP total (\%) \\
\hline 2002 & 1383 & $29(2)$ & $27(2)$ & $4(0.3)$ & $5(0.4)$ \\
2003 & 1206 & $23(2)$ & $29(2.5)$ & $14(1)$ & $50(4.3)$ \\
2004 & 1231 & $36(3)$ & $39(3)$ & $9(0.8)$ & $57(5)$ \\
2005 & 1147 & $39(3.4)$ & $45(4)$ & $3(0.3)$ & $7(0.7)$ \\
2006 & 1036 & $41(4)$ & $26(2.5)$ & 42 & $70(8)$ \\
2007 & 994 & $49(5)$ & $18(1.8)$ & 184 & $74(7.4)$ \\
Total & 6997 & 217 & 7440 \\
\hline
\end{tabular}

$I A B P$, Intraaortic balloon pump. Test for difference in numbers and timing of insertion between years: Pearson chi-square $(15)=47.4214, P<.001$.

pulmonary hypertension, and unstable angina remain statistically significant.

Figure 1 shows postoperative length of stay in the groups in the sample stratified by timing of IABP insertion. This graph shows a substantially skewed distribution of length of stay and early deaths resulting in a shorter length of stay. Overall, the postoperative group had the longest ventilation times and ICU and hospital stays. The preoperative group had a lower median duration of ventilation (24 vs 36 hours), lower ICU stay (69 vs 90 hours), and borderline lower postoperative hospital length of stay (10 vs 12 days) compared with the intraoperative group. When allowance was made for multiple comparisons, the preoperative group showed statistically significantly shorter ICU hours than the intraoperative group.

Table 5 shows the predictors of use of an intraoperative or postoperative IABP using preoperative factors and excluding those who had a preoperative IABP. Table 5 shows that in addition to well-described risk factors (poor ejection fraction, reoperation, and unstable angina), recent myocardial infarction, renal dysfunction, pulmonary hypertension, and complex surgery were associated with the requirement for an intraoperative or postoperative IABP.

\section{DISCUSSION}

This study of 7440 cardiac surgical patients showed increasing use of IABP and a trend to preoperative insertion over time. It also demonstrated a lower crude mortality with a trend to lower risk-adjusted mortality after preopera- tive compared with intraoperative insertion of IABP. The findings were affected by the risk-adjustment method used and confounded by the duration of cardiopulmonary bypass time. The group that required a postoperative IABP had a higher than expected mortality regardless of risk adjustment method.

The strength of our study is that the number of patients evaluated is large. Data were collected prospectively using standardized forms by trained personnel. Outcome measures were clearly defined (in-hospital mortality, hospital and ICU length of stay, ventilation hours, and complications). Logistic EuroSCORE was used to predict the risk of death and compared with the observed mortality.

The limitation of this study is that it is not a randomized study but a single-center retrospective analysis of prospectively collected data. Potential confounding may also exist, which is difficult to control for in such a study. However, in keeping with the previous research in this area, we have attempted to control for the confounding by comparing the observed mortality with predicted risk of death from the EuroSCORE. It may be suggested that the IABP itself, rather than the disease process, dictates the emergency of the procedure, rather than the disease per se. However, detailed examination of our data indicates that the patients receiving a preoperative balloon were indeed sicker, as indicated by a higher number fulfilling the critical preoperative state criteria and having a significantly higher logistic risk of death compared with intraoperative or postoperative groups, regardless of IABP status.

TABLE 3. Relationship between timing of intraaortic balloon pump insertion, operative characteristics, and outcomes

\begin{tabular}{|c|c|c|c|c|}
\hline & No IABP & Preoperative & Intraoperative & Postoperative \\
\hline \multicolumn{5}{|l|}{ Intraoperative variables } \\
\hline Median CPB time with IQR (min)* & $* 85(67-112)$ & $93(70-126)$ & $134(105-188)$ & $114(81-167)$ \\
\hline \multicolumn{5}{|l|}{ Outcomes } \\
\hline In-hospital mortality (\%) & $59(0.8)$ & $21(10)$ & $29(16)$ & $12(29)$ \\
\hline Median ventilation hours (IQR) $\dagger$ & $\dagger 9(7-14)$ & $24(17-68)$ & $36(21-88)$ & $99(35-214)$ \\
\hline Median ICU LOS hours (IQR) $\dagger$ & $24(21-42)$ & $69(45-120)$ & $90(62-152)$ & $155(74-447)$ \\
\hline Reventilation (\%) & $201(3)$ & $19(9)$ & $18(10)$ & $11(26)$ \\
\hline $\begin{array}{l}\text { Median postoperative hospital } \\
\text { LOS days (IQR) }\end{array}$ & $8(7-10)$ & $10(8-18)$ & $12(9-19)$ & $17(10-36)$ \\
\hline
\end{tabular}


TABLE 4. Relationship between timing of intraaortic balloon pump insertion and in-hospital mortality

\begin{tabular}{|c|c|c|c|c|}
\hline & Univariate & $\begin{array}{l}\text { Multivariate adjusting } \\
\text { for EuroSCORE }\end{array}$ & $\begin{array}{c}\text { Multivariate using } \\
\text { preoperative variables }\end{array}$ & $\begin{array}{l}\text { Multivariate using preoperative variables and with } \\
\text { adjustment for cardiopulmonary bypass time }\end{array}$ \\
\hline No IABP & 1 & 1 & 1 & 1 \\
\hline Preoperative & $12.6(7.5-21)$ & $3.9(2.1-7.1)$ & $5.9(3-12)$ & $5(2.5-10)$ \\
\hline Intraoperative & $22(13.7-55)$ & $12.6(7.5-21.1)$ & $11.5(6.7-20)$ & $6.5(3.6-12)$ \\
\hline Postoperative & $47(23-96)$ & $30.4(14.4-68)$ & $26(11-60)$ & $22(9-52)$ \\
\hline \multicolumn{5}{|l|}{ Model properties } \\
\hline $\begin{array}{l}\text { Hosmer-Lemeshow } \\
\text { goodness-of-fit }\end{array}$ & N/A & $P=.01$ & $P=.48$ & $P=.23$ \\
\hline ROC & 0.73 & 0.9 & 0.9 & 0.91 \\
\hline
\end{tabular}

EuroSCORE, European System for Cardiac Operative Risk Evaluation; IABP, intraaortic balloon pump; ROC, receiver operating characteristic; N/A, not available. Cells show odds ratio and $95 \%$ confidence interval (in brackets). No IABP insertion is the reference group unless otherwise specified. Preoperative variables included were age, gender, vascular disease, reoperation, chronic obstructive pulmonary disorder, neurologic dysfunction, ejection fraction $<30 \%$, ejection fraction $30 \%-50 \%$, serum creatinine, complexity of surgery, and preoperative mechanical ventilation. The Hosmer-Lemeshow goodness-of-fit test was not appropriate for the univariate analysis because there were only 2 degrees of freedom.

A recent meta-analysis found that preoperative IABP deployment may have a beneficial effect in specific high-risk groups undergoing CABG. ${ }^{13}$ The randomized control trials included in the meta-analysis had small treatment group numbers (range, 30-60 patients) and notably were performed in a single institution. ${ }^{14-18}$

Several nonrandomized studies have shown a survival benefit with preoperative IABPs, especially with "off pump" CABG. ${ }^{19-21}$ However, all these studies had included a higher number of unstable high-risk patients in the intervention arm, and "no difference in mortality" between the 2 groups was taken as a survival benefit in the intervention group. Of the "on pump" studies, a few retrospective studies have found preoperative IABPs useful in high-risk patients. $^{22,23}$ However, the intervention groups in these studies had a higher number of unstable patients and patients with unstable angina or recent myocardial infarction. Another large retrospective study that compared stable with unstable high-risk patients found that preoperative insertion of

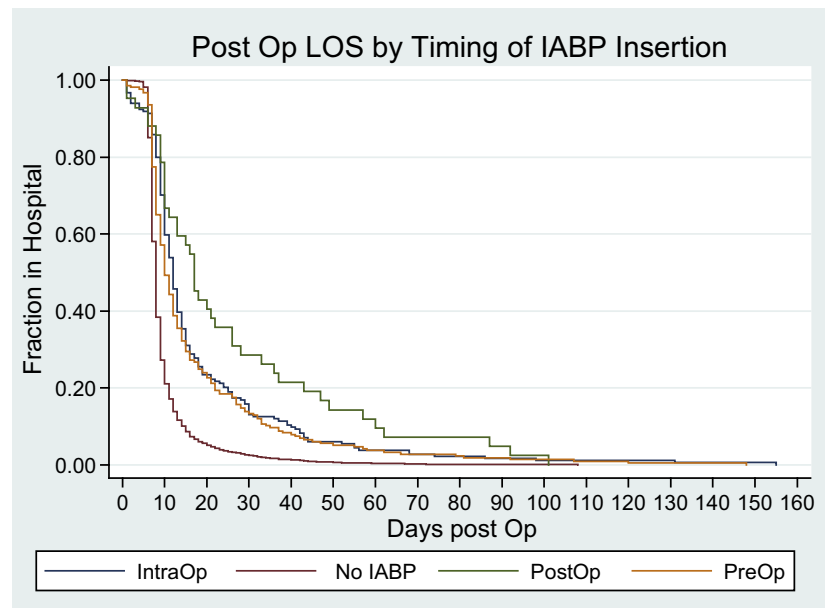

FIGURE 1. Time to hospital discharge by timing of IABP insertion. IABP, Intraaortic balloon pump; $L O S$, length of stay.
IABP conferred no survival benefit but was associated with a reduced hospital length of stay. ${ }^{24}$.

A recent meta-analysis that included randomized trials and cohort studies has supported the use of preoperative IABPs in high-risk patients in terms of mortality benefit and reduced ICU length of stay. ${ }^{9}$ Dyub and colleagues' study ${ }^{9}$ found an increased hospital and ICU length of stay with IABP use, especially in the postoperative group, which conflicts with some previous reports. ${ }^{25,26}$ Differences in the patient selection criteria in the included studies may account for the result. Other possible reasons may be the increased severity of illness in this patient group and also the IABP itself. The need to remain supine with an IABP predisposes patients to atelectasis, pneumonia, and lower limb venous thromboses. This review also found that the risk of complications (limb ischemia or hematoma) was low and reversible with discontinuation and removal of the balloon $(3.7 \%)$. Of note, most included studies mentioned the use of $8 \mathrm{~F}$ or larger catheters. Therefore, there is potential to reduce complication rates further by using smaller-caliber catheters and sheathless placement techniques. In our hospital, only

TABLE 5. Predictors of use of intraoperative or postoperative intraaortic balloon pump in those who did not receive a preoperative intraaortic balloon pump

\begin{tabular}{lcrc}
\hline \multicolumn{1}{c}{ Variables } & Odds ratio $(\mathbf{9 5} \% \mathbf{C I})$ & \multicolumn{1}{c}{$\mathbf{Z}$} & $\boldsymbol{P}>|\mathbf{z}|$ \\
\hline Reoperation & $3.5(2.5-5)$ & 7.22 & .000 \\
Creatinine $>200 \mu \mathrm{mol} / \mathrm{L}$ & $2.5(1.4-4.4)$ & 3.24 & .001 \\
Unstable angina & $5.2(3.1-8.6)$ & 6.29 & .000 \\
EF $<30 \%$ & $9.5(6.3-14.5)$ & 10.53 & .000 \\
EF $30 \%-50 \%$ & $2.3(1.7-3.1)$ & 5.23 & .000 \\
Recent MI & $2.3(1.6-3.2)$ & 4.95 & .000 \\
Pulmonary hypertension & $1.9(1.3-3)$ & 3.00 & .003 \\
1 valve only & $0.6(0.3-1.1)$ & -1.68 & .093 \\
1 valve + CABG & $2.3(1.6-3.4)$ & 4.41 & .000 \\
Complex surgery & $2.3(1.6-3.5)$ & 4.12 & .000 \\
\hline
\end{tabular}

$C I$, Confidence interval; $E F$, ejection fraction; $M I$, myocardial infarction; $C A B G$, coronary artery bypass grafting. 
catheters $7.5 \mathrm{~F}$ and smaller are used now. Indeed, female patients (who predominantly received intraoperative or postoperative IABPs) may perhaps benefit from having a smaller sheathless catheter placed preoperatively, given that female gender is a known risk factor for increased mortality with CABG.

The postoperative group in our study had an in-hospital mortality that, although comparable to the mortality rates described in similar studies, was higher than other groups in our study. This group requires further evaluation to determine whether an earlier or preoperative IABP placement would improve outcomes. It is possible that this group experienced complications not recognized in the operative room.

The major disadvantages to IABP use thus far have been complications associated with its placement, mainly bleeding, vascular injury, and limb ischemia. ${ }^{11}$ Availability of smallercaliber 7F catheters and sheathless insertion techniques has considerably reduced the incidence of vascular complications. Furthermore, advances in IABP design, such as fiberoptic technology, which provides higher fidelity arterial pressure waveforms, elimination of need for repeated zeroing, and automatic trigger modes, have made day-to-day handling of balloon pumps simpler, more accurate, and user-friendly.

This study also showed that it is possible to predict which patients may require an intraoperative or a postoperative balloon pump. This information may be useful in a long overdue randomized trial of the timing of IABP insertion. Randomized studies are needed to further delineate groups who would most benefit from preoperative IABPs. Because the majority of randomized studies in this area are from a single institution, there is a need for an adequately powered multicenter study.

\section{CONCLUSIONS}

Preoperative IABP use was associated with a relative reduction in risk-adjusted mortality in this study. Our study therefore supports the use of preoperative IABP in carefully selected patients. Predictors of the need for an intraoperative or a postoperative IABP were developed using simple, widely available preoperative variables. This may assist in the planning of a randomized study of preemptive IABP use in high-risk patients.

\section{References}

1. Cresswell LL, Rosenbloom M, Cox JL, Ferguson TB Sr, Kouchoukas NT, Spray TL, et al. Intraaortic balloon counterpulsation: patterns of usage and outcome in cardiac surgery patients. Ann Thorac Surg. 1992;54:11-20.

2. Nauheim KS, Swartz MT, Pennington DG, Fiore AC, McBride LR, Peigh PS, et al. Intraaortic balloon pumping in patients requiring cardiac operations: risk analysis and long term follow-up. J Thorac Cardiovasc Surg. 1992;104:1654-61.

3. Ferguson JJ III, Cohen M, Freedman RJ Jr, Stone GW, Miller MF, Joseph DL, et al. The current practice of intra-aortic balloon counterpulsation: results from the Benchmark Registry. J Am Coll Cardiol. 2001;38:1456-62.

4. Christenson JT, Cohen M, Ferguson JJ III, Freedman RJ, Miller MF, Ohman EM et al. Trends in intraaortic balloon counterpulsation complications and outcomes in cardiac surgery. Ann Thorac Surg. 2002;74:1086-90.
5. Cohen M, Urban P, Christenson JT, Joseph DL, Freedman RJ Jr, Miller MF, et al. Benchmark Registry Collaborators. Intra-aortic balloon counterpulsation in US and non-US centres: results of the Benchmark Registry. Eur Heart J. 2003;24: 1763-70.

6. Lewis PA, Mullany DV, Townsend S, Johnson J, Wood L, Courtney MD, et al. Trends in intra-aortic balloon counterpulsation: comparison of a 669 record Australian dataset with the multinational Benchmark Counterpulsation Outcomes Registry. Anaesth Intensive Care. 2007;35:13-9.

7. Lewis PA, Mullany DV, Courtney M, Coyer F. Australian trends in intra-aortic balloon counterpulsation weaning: results of a postal survey. Crit Care Resusc. 2006;8:361-7.

8. Yap C, Reid C, Yii M, Rowland M, Mohajiri M, Skillington P, et al. Validation of the EuroSCORE model in Australia. Eur J Cardiothorac Surg. 2006;29:441-6.

9. Dyub AM, Whitlock RP, Abouzahr LL, Cina CS. Preoperative Intraaortic balloon pump in patients undergoing coronary bypass surgery: a systematic review and meta-analysis. J Card Surg. 2008;23:79-86.

10. Marra C, De Santo LS, Amarelli C, Dellacorte A, Onorati F, Torella M, et al. Coronary artery bypass grafting in patients with severe left ventricular dysfunction: a prospective randomized study on the timing of perioperative intraaortic balloon pump support. Int J Artif Organs. 2002;25:141-6.

11. Meharwal ZS, Trehan N. Vascular complications of intra-aortic balloon insertion in patients undergoing coronary revascularization: analysis of 911 cases. Eur $J$ Cardiothorac Surg. 2002;21:741-7.

12. Nashef SA, Roques F, Gauducheau E, Lemeshow S, Salamon R. European system for cardiac operative risk evaluation (EuroSCORE). Eur J Cardiothorac Surg. 1999;16:9-13.

13. Field ML, Rengarajan A, Khan O, Spyt T, Richens D. Preoperative intraaortic balloon pumps in patients undergoing coronary artery bypass grafting. Cochrane Database Syst Rev. 2007;1.

14. Christenson JT, Simonet F, Badel P, Schmuziger M. The effect of preoperative intra-aortic balloon pump support in patients with coronary artery disease, poor left ventricular function (LVEF $<40 \%$ ) and hypertensive LV hypertrophy. Thorac Cardiovasc Surg. 1997;45:60-4.

15. Christenson JT, Simonet F, Badel P, Schmuziger M. Preoperative intraaortic balloon pump enhances cardiac performance and improves the outcome of redo CABG. Ann Thorac Surg. 1997;64:1237-44.

16. Christenson JT, Simonet F, Badel P, Schmuziger M. Evaluation of preoperative intraaortic balloon pump support in high risk coronary patients. Eur J Cardiothorac Surg. 1997;11:1097-103.

17. Christenson JT, Simonet F, Badel P, Schmuziger M. Optimal timing of preoperative intraaortic balloon pump support in high risk coronary patients. Ann Thorac Surg. 1999;68:934-9.

18. Christenson JT, Liker M, Kalangos A. The role of intraaortic counterpulsation in high risk OPCAB surgery: a prospective randomized study. J Card Surg. 2003;18: 286-94.

19. Suzuki T, Okabe M, Handa M, Yasuda F, Miyake Y. Usefulness of preoperative IABP therapy during off-pump coronary artery bypass grafting in high risk patients. Ann Thorac Surg. 2004;77:2056-9.

20. Kim KB, Lim C, Ahn H, Yang JK. Intraaortic balloon pump therapy facilitates posterior vessel off-pump coronary artery bypass grafting in high risk patients. Ann Thorac Surg. 2001;71:1964-8.

21. Craver JM, Murrah CP. Elective intraaortic balloon counterpulsation for high-risk off-pump coronary artery bypass operations. Ann Thorac Surg. 2001;71:1220-3.

22. Gutfinger DE, Ott RA, Miller M, Selvan A, Codini M, Alimadadian H, et al. Aggressive preoperative use of intraaortic balloon pump in elderly patients undergoing coronary artery bypasses grafting. Ann Thorac Surg. 1999;67: 610-3.

23. Dietl CA, Berkheimer MD, Woods EL, Gibert CL, Pharr WF, Benoit CH. Efficacy and cost effectiveness of preoperative IABP in patients with ejection fraction of 0.25 or less. Ann Thorac Surg. 1996;62:401-9.

24. Holman W, Qing L, Kiefe C, McGiffin D, Peterson E, Allman R, et al. Prophylactic value of preincision intraaortic balloon pump: analysis of a state wide experience. J Thorac Cardiovasc Surg. 2000;120:1112-9.

25. Christenson JT, Simonet F, Schmuziger M. Economic impact of preoperative intraaortic balloon pump therapy in high-risk coronary patients. Ann Thorac Surg. 2000;70:510-5.

26. Talley M, Ohman M, Mark M, George BS, Leimberger JD, Berdan LG, et al. Economic implications of the prophylactic use of intraaortic balloon counterpulsation in the setting of acute myocardial infarction. Am J Cardiol. 1997;79:590-4. 